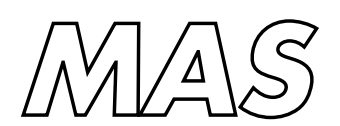

Modelling, Analysis and Simulation

Modelling, Analysis and Simulation
MAS A note on flux limiting for diffusion discretizations

W. Hundsdorfer, C. Montijn

Report MAS-E0305 August 31, 2003 
CWI is the National Research Institute for Mathematics and Computer Science. It is sponsored by the Netherlands Organization for Scientific Research (NWO).

CWI is a founding member of ERCIM, the European Research Consortium for Informatics and Mathematics.

CWI's research has a theme-oriented structure and is grouped into four clusters. Listed below are the names of the clusters and in parentheses their acronyms.

Probability, Networks and Algorithms (PNA)

Software Engineering (SEN)

Modelling, Analysis and Simulation (MAS)

Information Systems (INS)

Copyright (C) 2003, Stichting Centrum voor Wiskunde en Informatica

P.O. Box 94079, 1090 GB Amsterdam (NL)

Kruislaan 413, 1098 SJ Amsterdam (NL)

Telephone +31205929333

Telefax +31205924199

ISSN 1386-3703 


\title{
A Note on Flux Limiting for Diffusion Discretizations
}

\author{
Willem Hundsdorfer, Carolynne Montijn \\ $C W I$
}

P.O. Box 94079, 1090 GB Amsterdam, The Netherlands

\begin{abstract}
In this note a limiting technique is presented to enforce monotonicity for higherorder spatial diffusion discretizations. The aim is to avoid spurious oscillations and to improve the qualitative behaviour on coarse grids. The technique is related to known ones for convection equations, using limiters to bound the numerical fluxes. Applications arise in pattern formation problems for reaction-diffusion equations.
\end{abstract}

2000 Mathematics Subject Classification: 65M06, 65M20.

Keywords and Phrases: Diffusion schemes, flux limiting, monotonicity, positivity. Note: Work carried out within the theme MAS3 "Nonlinear Dynamics and Complex Systems". The work of C. Montijn is supported by an NWO grant within the Computational Science programme.

\section{Introduction}

The preservation of monotonicity properties - like positivity, maximum principles and comparison principles - is often essential for numerical schemes to approximate nonsmooth solutions in a qualitatively correct manner. For convection equations this has led to the development of 'high-resolution' schemes with flux limiters. The object of this note is to present a similar technique for diffusion equations. Applications are found in pattern formation problems for reaction-diffusion equations where patterns are formed by intrusion of fronts into unstable steady states, the so-called pulled front problems in the terminology of [1].

Consider the reaction-diffusion equation

$$
u_{t}=u_{x x}+g(u)
$$

for $t \geq 0, x \in \mathbb{R}$, with initial condition $u(x, 0)$ given. We will deal with spatial discretizations on uniform grids with mesh width $h>0$, grid points $x_{j}=x_{0}+j h$ and surrounding cells $\Omega_{j}=\left[x_{j}-\frac{1}{2} h, x_{j}+\frac{1}{2} h\right]$. In the resulting semi-discrete system $w_{j}(t)$ stands for an approximation to the point value $u\left(x_{j}, t\right)$ or the cell-average value on $\Omega_{j}$ at time $t$. We will mostly denote $w_{j}=w_{j}(t), w_{j}^{\prime}=w_{j}^{\prime}(t)$, thus omitting the temporal arguments.

Spatial discretization in conservation form leads to

$$
w_{j}^{\prime}=\frac{1}{h}\left(f_{j-\frac{1}{2}}-f_{j+\frac{1}{2}}\right)+g\left(w_{j}\right)
$$

with diffusive fluxes $f_{j \pm 1 / 2}$ at the cell boundaries, located half-way between the grid points $x_{j}$. Standard choices are

$$
f_{j+\frac{1}{2}}=\frac{1}{h}\left(w_{j}-w_{j+1}\right),
$$


giving the usual second-order discretization, and

$$
f_{j+\frac{1}{2}}=\frac{1}{12 h}\left(-w_{j-1}+15 w_{j}-15 w_{j+1}+w_{j+2}\right),
$$

for the fourth-order discretization. The latter discretization is known to produce some oscillations and associated unphysical negative solution values if the exact solution, viewed as a grid function, is not smooth relative to the mesh width $h$. This is caused by the fact that the resulting semi-discrete system $w^{\prime}=A w+g(w)$ will have a matrix $A$ with negative off-diagonal elements; see [3], for instance.

In this note we propose a novel spatial discretization scheme, which is obtained by limiting the fourth-order flux so as to enforce monotonicity. The technique used here is similar to discretizations for convection terms that are well-known and often used for hyperbolic conservation laws; see for example $[3,4,5]$. The discretization scheme will be tested on the Fisher equation.

\section{Limiting of Diffusive Fluxes}

We consider a 'limited form' of the fourth-order discretization. In the following, let $\bar{\jmath}=j+\frac{1}{2}$ and

$$
v_{\bar{\jmath}}=\frac{1}{h}\left(w_{j}-w_{j+1}\right), \quad \theta_{\bar{\jmath}}=\frac{v_{\bar{\jmath}-1}-v_{\bar{\jmath}}}{v_{\bar{\jmath}}-v_{\bar{\jmath}+1}}=\frac{w_{j-1}-2 w_{j}+w_{j+1}}{w_{j}-2 w_{j+1}+w_{j+2}} .
$$

Then the fourth-order formula can also be written as

$$
f_{\bar{\jmath}}=v_{\bar{\jmath}}-\frac{1}{12}\left(v_{\bar{\jmath}-1}-2 v_{\bar{\jmath}}+v_{\bar{\jmath}+1}\right)=v_{\bar{\jmath}}-\frac{1}{12}\left(\theta_{\bar{\jmath}}-1\right)\left(v_{\bar{\jmath}}-v_{\bar{\jmath}+1}\right) .
$$

We will consider the more general formula

$$
f_{\bar{\jmath}}=v_{\bar{\jmath}}-\phi\left(\theta_{\bar{\jmath}}\right)\left(v_{\bar{\jmath}}-v_{\bar{\jmath}+1}\right)=v_{\bar{\jmath}}-\frac{1}{\theta_{\bar{\jmath}}} \phi\left(\theta_{\bar{\jmath}}\right)\left(v_{\bar{\jmath}-1}-v_{\bar{\jmath}}\right) .
$$

Here the function $\phi$ will be used to limit the fluxes. Of course, with $\phi=0$ we simply recover the second-order fluxes, whereas the choice $\phi(\theta)=\frac{1}{12}(\theta-1)$ corresponds to the fourth-order fluxes. Note that in smooth regions, with non-vanishing spatial second derivative, we will have $\theta_{\bar{\jmath}}=1+\mathcal{O}(h)$. Further, since the diffusion equation is symmetric in space, it is natural to impose this also on the discretization. As we will see shortly, this is achieved by

$$
\frac{1}{\theta} \phi(\theta)=-\phi\left(\frac{1}{\theta}\right) .
$$

Let us first consider the discretization (1.2) without the source term $g$. Then we have

$$
w_{j}^{\prime}=\frac{1}{h}\left(f_{j-\frac{1}{2}}-f_{j+\frac{1}{2}}\right)=\frac{1}{h}\left(v_{\bar{\jmath}-1}-\phi\left(\theta_{\bar{\jmath}-1}\right)\left(v_{\bar{\jmath}-1}-v_{\bar{\jmath}}\right)-v_{\bar{\jmath}}+\frac{1}{\theta_{\bar{\jmath}}} \phi\left(\theta_{\bar{\jmath}}\right)\left(v_{\bar{\jmath}-1}-v_{\bar{\jmath}}\right)\right) .
$$

Since $h\left(v_{\bar{\jmath}-1}-v_{\bar{\jmath}}\right)=w_{j-1}-2 w_{j}+w_{j+1}$, this can be written as

$$
w_{j}^{\prime}=\frac{1}{h^{2}} d_{j}\left(w_{j-1}-2 w_{j}+w_{j+1}\right)
$$

with

$$
d_{j}=1-\phi\left(\theta_{\bar{\jmath}-1}\right)+\frac{1}{\theta_{\bar{\jmath}}} \phi\left(\theta_{\bar{\jmath}}\right)
$$


The function $\phi$ will be limited such that

$$
-\mu_{0} \leq \phi(\theta) \leq \mu_{1}, \quad-\mu_{1} \leq \frac{1}{\theta} \phi(\theta) \leq \mu_{0}
$$

for all $\theta \in \mathbb{R}$. In these constraints the condition (2.3) is already incorporated, and it is easily seen from (2.4) that this condition gives symmetry in the discretization. Within these constraints $(2.5)$ we want to retrieve the fourth-order flux, $\phi(\theta)=\frac{1}{12}(\theta-1)$, as much as possible and certainly near $\theta=1$. A possible choice is shown in Figure 1 .

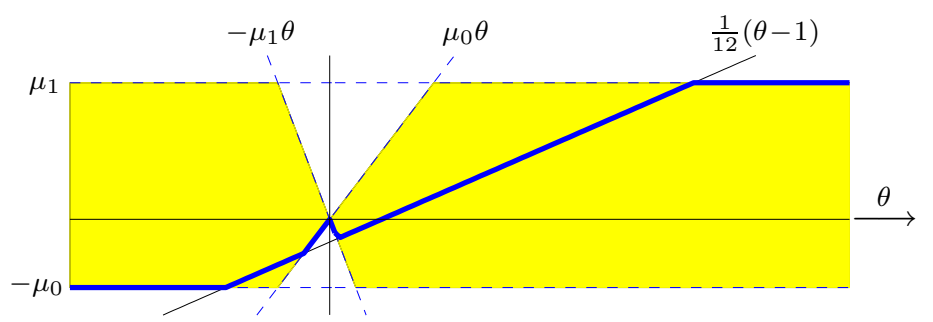

Figure 1: Monotonicity region (2.5) (shaded) and limiter function $\phi(\theta)$ as in (2.7).

The limiter parameters $\mu_{0}, \mu_{1}>0$ are chosen such that we will have monotonicity. In particular we will take

$$
\mu_{1} \leq \frac{1}{2}
$$

Note that (2.4) then can be written in the quasi-linear form $w^{\prime}=A(w) w$, where the off-diagonal elements of the matrix $A(w)$ are non-negative. As we will see below, this guarantees monotonicity of the discretization, and the choice of $\mu_{0}$ will determine the maximal time step that can be taken by ODE methods.

In the tests we will take $\mu_{1}=\frac{1}{2}, \mu_{0}=\frac{1}{4}$. The choice of $\mu_{0}$ turned out to have little significance on the quality of the spatial discretization in the tests below. The full formula for the limiter used in this paper is

$$
\phi(\theta)=\min \left(\mu_{1}, \max \left(\chi(\theta), \frac{1}{12}(\theta-1)\right)\right),
$$

where

$$
\chi(\theta)=\max \left(-\mu_{0}, \min \left(\mu_{0} \theta,-\mu_{1} \theta\right)\right) .
$$

This limiter is illustrated by the thick line in Figure 1. In the implementation we calculate the limited fluxes by the first equality in (2.2), where a very small number is added to the denominators of the ratios $\theta_{\bar{\jmath}}$ to avoid division by 0 .

\section{Monotonicity}

The above semi-discrete system reads in vector form

$$
w^{\prime}(t)=A(w(t)) w(t)+g(w(t)),
$$

with a matrix $A(w)=\left(a_{j k}(w)\right)$ whose elements are given by

$$
a_{j j}(w)=-\frac{2}{h^{2}} d_{j}(w), \quad a_{j, j \pm 1}(w)=\frac{1}{h^{2}} d_{j}(w)
$$


in terms of the coefficients $d_{j}=d_{j}(w)$ from $(2.4 \mathrm{~b})$, and where the reaction-source term is to be interpreted component-wise. The constraints (2.5), (2.6) imply that

$$
0 \leq 1-2 \mu_{1} \leq d_{j}(w) \leq 1+2 \mu_{0} .
$$

To discuss monotonicity, it is convenient to invoke temporal discretizations. Let $t_{n}=n \tau$ with step size $\tau>0$, and let us denote $w_{n}=\left(w_{j}^{n}\right)$ for the approximations to $w\left(t_{n}\right)=\left(w_{j}\left(t_{n}\right)\right)$. The forward Euler method gives

$$
w_{n+1}=\left(I+\tau A\left(w_{n}\right)\right) w_{n}+\tau g\left(w_{n}\right) .
$$

We see that

$$
I+\tau A\left(w_{n}\right) \geq 0 \quad \text { and } \quad\left\|I+\tau A\left(w_{n}\right)\right\|_{\infty} \leq 1
$$

provided that $\mu_{1} \leq \frac{1}{2}$ and the time step is restricted by

$$
\tau \leq \frac{h^{2}}{2\left(1+2 \mu_{0}\right)} .
$$

Here, inequalities for matrices or vectors are component-wise. Hence, if $g=0$, we have $\left\|w_{n}\right\|_{\infty} \leq\left\|w_{0}\right\|_{\infty}$ under this time step restriction, and likewise $w_{n} \geq 0$ if $w_{0} \geq 0$.

With a non-zero source term $g$ the positivity property $w_{n} \geq 0$ remains valid if $g(v) \geq 0$ for all scalar $v \geq 0$. The addition of source terms may also lead to extra time step restrictions. For example, if we assume

$$
\left\|I+\tau A\left(w_{n}\right)\right\|_{\infty} \leq 1 \quad \text { for } \tau \leq \tau_{0}, \quad\left\|w_{n}+\tau g\left(w_{n}\right)\right\|_{\infty} \leq\left\|w_{n}\right\|_{\infty} \quad \text { for } \tau \leq \tau_{1},
$$

then it can be seen that

$$
\left\|w_{n}+\tau A\left(w_{n}\right) w_{n}+\tau g\left(w_{n}\right)\right\|_{\infty} \leq\left\|w_{n}\right\|_{\infty} \quad \text { for } \tau \leq \frac{\tau_{0} \tau_{1}}{\tau_{0}+\tau_{1}} .
$$

This implication easily follows by writing the left-hand side as

$$
\left\|\kappa\left(w_{n}+\frac{\tau}{\kappa} A\left(w_{n}\right) w_{n}\right)+(1-\kappa)\left(w_{n}+\frac{\tau}{1-\kappa} g\left(w_{n}\right)\right)\right\|_{\infty}
$$

with $\kappa=\tau_{1} /\left(\tau_{0}+\tau_{1}\right)$. Related monotonicity results, like maximum principles, can be obtained in a similar way.

With such monotonicity results for the forward Euler method one can then derive similar results for higher-order schemes, including implicit ones; see for example [2, 3]. Here it should be noted that also for implicit methods, except for the backward Euler method, time step restrictions will arise. This is in contrast to the usual linear stability analysis by Fourier decompositions (von Neumann analysis), where $A$-stable time stepping methods yield unconditional stability. These results are completely similar to those for convection equations found in the above citations.

Finally we note that for the limited discretization the allowable time step is governed by $\mu_{0}$ as in (3.5). We will take $\mu_{0}=\frac{1}{4}$, and this gives a slight decrease in allowable step sizes compared to the standard second-order scheme, which corresponds to $\mu_{0}=0$.

Remark. For equations $u_{t}=\left(D(x, u) u_{x}\right)_{x}+g(x, u)$ with variable or nonlinear diffusion coefficients, the above fluxes $f_{j+1 / 2}$ are to be multiplied by $D_{j+1 / 2}$, approximating the coefficient at the cell boundaries. The limiter will then have to be adjusted to cope with the variations in the coefficients. Note that due to the conservation form (1.2) of the discretizations, mass conservation of the schemes is always ensured. 
Alternative forms are possible. For example, let $f_{j+1 / 2}^{*}=f_{\bar{\jmath}}^{*}$ be any non-limited flux, then define $\phi_{\bar{j}}^{*}$ by the relation

$$
\phi_{\bar{\jmath}}^{*}=-\frac{f_{\bar{\jmath}}^{*}-v_{\bar{\jmath}}}{v_{\bar{\jmath}}-v_{\bar{\jmath}+1}},
$$

and then apply limiting as before by (2.7) with $\theta=\theta_{\bar{\jmath}}$, where now $\phi_{\bar{\jmath}}^{*}$ replaces the original expression $\frac{1}{12}\left(\theta_{\bar{\jmath}}-1\right)$. This formulation applies also to higher-order non-limited fluxes.

Finally we note that for multi-dimensional problems on Cartesian grids the above results carry over directly by applying limiting in the various spatial directions.

\section{Numerical Illustration for the Fisher Equation}

The Fisher equation is a prototype for many pattern formation problems. The equation reads

$$
u_{t}=\varepsilon u_{x x}+\gamma u\left(1-u^{2}\right) .
$$

An exact traveling wave solution is given by

$$
u(x, t)=\left(1+e^{\lambda(x-1-\alpha t)}\right)^{-1}
$$

with $\lambda=\frac{1}{2} \sqrt{2 \gamma / \varepsilon}$ and $\alpha=\frac{3}{2} \sqrt{2 \gamma \varepsilon}$. In the following tests we consider parameter values $\gamma=\varepsilon^{-1}=100$. Further $0<x<L=6$ and $0<t \leq T=1$. The initial condition at $t=0$ is taken according to (4.2). At the boundaries homogeneous Neumann conditions are imposed. This gives a close approximation to the exact $u_{x}$ at the boundaries provided the end time $T$ is not too large, so that the wave does not reach the boundary.

The temporal discretization is performed with a high-order explicit Runge-Kutta method using small time steps. The numerical errors in the following figures are solely due to the spatial discretization. The exact solution at time $T=1$ and the corresponding numerical results with $h=L / 120$ are given in Figure 2 .
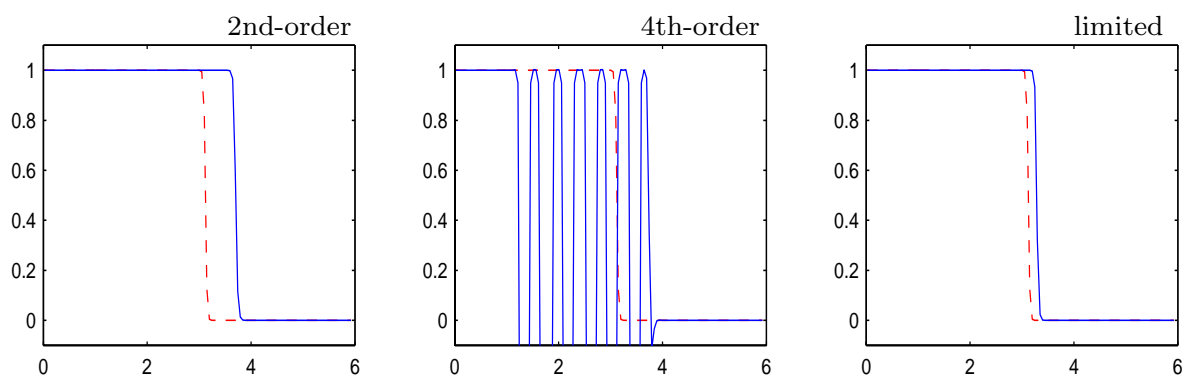

Figure 2: Numerical solutions with $h=L / 120=1 / 20$ for the second-order scheme (left), the fourth-order scheme (middle) and the limited scheme (right). The exact solution is indicated by the dashed lines.

Obviously the wave speed is incorrect for the second-order scheme. This is due to the fact that we are dealing here with a so-called 'pulled front' problem, where the dynamics are determined to a large extent by the solution ahead of the front, see [1]. The asymptotically small values ahead of the front are important for the wave speed and apparently these are not captured with enough precision on this grid by the second-order numerical discretization. 
The fourth-order scheme produces qualitatively wrong solutions. Due to the lack of monotonicity of the diffusion discretization, negative solution values are created, which are then amplified towards -1 by the reaction term. The limited scheme provides a good compromise. By the limiting the oscillations have disappeared, but in the flat region ahead of the front the fourth-order scheme will still be used, leading to a more accurate front velocity than with the second-order scheme.

Results for the discretizations on various grids are given in Table 1. The entries are the absolute $L_{1}$-errors at output time $T=1$. These results are in line with those obtained for $h=L / 120$. We see that the fourth-order scheme needs a fine grid to produce a reasonable solution, but then it is much more accurate than the second-order scheme. The limited scheme combines the advantages of the two standard discretizations: it gives monotone and reasonable solutions on coarse grids and on fine grids it gives approximately the same errors as the fourth-order scheme.

\begin{tabular}{l||c|c|c|c|c|c}
$m=L / h$ & 30 & 60 & 120 & 240 & 480 & 960 \\
\hline \hline 2nd-order & 2.7085 & 1.4104 & 0.5865 & 0.1782 & 0.0439 & 0.01087 \\
4th-order & 4.6403 & 4.0312 & 2.6766 & 0.2460 & 0.0045 & 0.00027 \\
Limited & 2.3276 & 0.9290 & 0.1753 & 0.0872 & 0.0046 & 0.00028 \\
\hline
\end{tabular}

Table 1: Absolute spatial $L_{1}$-errors for the Fisher equation.

Remark. In the present test the form (2.2) was used. In CPU time, for a vectorized MATLAB program, the limited scheme was here roughly a factor 1.8 more expensive than the second-order scheme and a factor 1.4 more expensive than the fourth-order scheme.

\section{Concluding Remarks}

The above limited spatial discretization scheme provides a suitable balance between the second-order and fourth-order schemes. On fine grids it gives approximately the same errors as the fourth-order scheme, whereas on coarse grids (relative to the smoothness of the solution) it gives monotone approximations with better accuracy than the standard second-order discretization.

Additional tests were carried out for related 1D problems like $u_{t}=\varepsilon u_{x x}+g(x, t)$ with source term $g(x, t)$ such that the solution is still given by (4.2). There the limited scheme produced results similar to the fourth-order scheme, and more accurate than the second-order scheme. It should be noted however that for such problems limiting is redundant, since small over- and undershoots will not lead to a wrong qualitative behaviour. The limiters then merely make the scheme computationally more expensive. Limiting should only be applied if necessary, of course.

It is only fair to say that for many reaction-diffusion problems violation of monotonicity will not be a problem. For typical combustion problems, for example, steep moving fronts are common, but there the states before and after the front are usually stable. Small over- and undershoots will then be quickly filled up. On the other hand, there is an interesting class of problems where lack of monotonicity will lead to a wrong qualitative behaviour. The Fisher equation is the standard example. For related problems, see [1, Sect. 5.6]. 
If a certain maximum principle is known a priori, then this might also be enforced of course by simply cutting-off numerical values outside the prescribed range. In this way, however, local mass balances might be destroyed. Moreover, detailed a priori information is usually not available.

In conclusion: the limiting procedure described here seems suited for reactiondiffusion problems where steep fronts enter spatial regions that are in an unstable equilibrium - the so-called 'pulled front' problems [1] - and where the mesh width is restricted by the computer capacity, as it often happens with 3D problems.

\section{References}

[1] U. Ebert, W. van Saarloos, Front propagation into unstable states: universal algebraic convergence towards uniformly translating pulled fronts. Physica D 146 (2000), 1-99.

[2] S. Gottlieb, C.-W. Shu, E. Tadmor, Strong stability preserving high-order time discretization methods. SIAM Review 42 (2001), 89-112.

[3] W. Hundsdorfer, J.G. Verwer, Numerical Solution of Time-Dependent AdvectionDiffusion-Reaction Equations. Springer Series in Computational Mathematics 33, Springer, Berlin, 2003.

[4] R.J. LeVeque, Finite Volume Methods for Hyperbolic Problems. Cambridge Texts in Applied Mathematics, Cambridge University Press, 2002.

[5] P.K. Sweby, High resolution schemes using flux-limiters for hyperbolic conservation laws. SIAM J. Numer. Anal. 21 (1984), 995-1011. 